\title{
Can Fixed Space Maintainers Have Adverse Effect on the Teeth and Periodontium?
}

\author{
Ahmad Behrooziann ${ }^{1}$, Parastou Nastarin², Marziyeh Aghazadeh ${ }^{3}$, Ahmad Pirzadeh Ashraf ${ }^{4}$, \\ Zahra Aghazadeh ${ }^{5}$, Mahtab Khojasteh Manesh ${ }^{6}$, Vali Alizadeh ${ }^{7}$
}

${ }^{1,2}$ Department of Orthodontics, Tabriz University of Medical Sciences, Tabriz, Iran, ${ }^{3}$ Stem Cell Research Centre and Department of Oral Medicine, Tabriz University of Medical Sciences, Tabriz,

Iran, ${ }^{4}$ Department of Endodontics, Tabriz Azad University, Tabriz, Iran, ${ }^{5}$ Department of Oral Medicine, Tabriz University of Medical Sciences, Tabriz, Iran, ${ }^{6}$ Private Practitioner, Shabestar, Iran, 7 Department of Community Dentistry, Tabriz Azad University, Tabriz, Iran.

\section{ABSTRACT}

\section{BACKGROUND}

Space maintenance after premature loss of primary teeth is of importance in preventing space loss and potential crowding in future. Fixed space maintainers are used to prevent space loss but the presence of such fixed appliances near the tooth and its supporting structures may have some adverse effects. The purpose of this study was to evaluate the potential adverse effects of fixed space maintainers on the teeth and periodontium.

\section{METHODS}

Twenty young children in mixed dentition period (between $8-11$ y $7 \mathrm{~m}$ years old) who were considered for space maintainer treatment were selected. Each patient had bilateral first molars that went under the band for fixed space maintainer. The patients were examined for decayed, missed and filled teeth (DMFT), bleeding on probing, clinical attachment level, gingival index and periodontal pocket depth at the beginning (T0), one month (T1), three months (T2) and six months (T3) later. Repeated measures ANOVA then Post - hoc LSD (Friedman test for gingival index) tests were used to interpret the data.

\section{RESULTS}

As compared to the beginning of the study, at the end of the 6 months follow-up period DMFT of the involved first molars did not change significantly $(P=0.163)$. But bleeding on probing, clinical attachment level, gingival index and periodontal pocket depths changed significantly at the same time frame $(\mathrm{P}<0.001)$.

\section{CONCLUSIONS}

The fixed space maintainers might have some adverse effects on the periodontal structures of the banded teeth, so the clinicians should insist more on oral hygiene instructions and the patients must be under intense oral hygiene control.

\section{KEY WORDS}

Space Maintainers, DMFT, Bleeding on Probing, Clinical Attachment Level, Periodontal Pocket Depth
Corresponding Author: Dr. Parastou Nastarin, Department of Orthodontics, Faculty of Dentistry, Tabriz University of Medical Sciences, Tabriz, Iran. E-mail:p.nastarin@gmail.com

\section{DOI: $10.14260 /$ jemds/2021/638}

How to Cite This Article:

Behroozian A, Nastarin P, Aghazadeh M, et al. Can fixed space maintainers have adverse effect on the teeth and periodontium? J Evolution Med Dent Sci 2021;10(36):3132-3136, DOI: $10.14260 /$ jemds/2021/638

Submission 13-03-2021,

Peer Review 30-07-2021,

Acceptance 06-08-2021,

Published 06-09-2021.

Copyright (C) 2021 Ahmad Behroozian et al. This is an open access article distributed under Creative Commons Attribution License [Attribution 4.0 International (CC $B Y 4.0)]$ 


\section{BACKGROUND}

Crowding and space deficiency in permanent dentition is the most common orthodontic problem. One of the major etiologic factors of permanent dentition crowding, is loss of space due to premature loss of deciduous teeth. ${ }^{1}$ Thus after the loss of deciduous teeth, arch length maintaining is mandatory during mixed dentition, which is accomplished by space maintainers (SM). ${ }^{2}$ SMs are classified into removable and fixed appliances; Unlike removable SMs, fixed SMs are independent of patient cooperation but need to maintain good oral hygiene. ${ }^{3}$ Previous studies have shown that fixed orthodontic appliances can cause more biofilm accumulation on teeth surfaces, ${ }^{4}$ hence there might be a higher prevalence of caries in presence of sugar consumption and under improper oral hygiene ${ }^{5,6}$, periodontium issues such as bleeding on probing, and increased pocket probing depth, which are more prominent when bands are used. ${ }^{7,8}$ In some studies it has been shown that orthodontic appliances increase the number of Streptococci mutans. ${ }^{9,10}$ This might increase the risk of caries occurrence in orthodontic patients. ${ }^{11}$

SMs are passive orthodontic appliances, and Arikan et al. showed that these appliances are related to increased plaque and biofilm accumulation on teeth which in turn can cause dental caries and periodontal problems. ${ }^{12}$ Arikan et al. in another study found that, both fixed and removable SMs could increase bleeding, plaque index and pocket depth scores. ${ }^{13}$ It is undisputed that SMs are effective in prevention of space loss but besides their benefits, their potential harms to the teeth and periodontium should also be assessed for best management of these appliances. To our knowledge by the time we were preparing the manuscript there was no published evidence addressing the effects of SMs on DMFT (the sum of Decayed, Missed and Filled teeth), so the objective of this study was to evaluate the effect of SMs on DMFT and also on periodontal status of patients, including gingival index, clinical attachment level, periodontal pocket depth and bleeding on probing during mixed dentition.

\section{METHODS}

The present in-vivo, before-after study was conducted at the Department of Community Dentistry and lasted for almost one year from 03-09-2015 to 29-09-2016. The research was reviewed and approved by ethics committee of the university. Twenty children with the age of $8-11.7$ years old, who required bilateral fixed space maintainers in their upper or lower jaws were selected amongst patients referred to this department according to inclusion criteria of this study. Therefore, total of 20 patients with 40 first permanent molars were included in this study by sequential sampling.

\section{Inclusion Criteria}

1. Patients in mixed dentition period and under 12 years old.

2. Being cooperative with the appliance,

3. At least one extracted posterior primary tooth.
4. Two millimetres or more of bone covering the permanent tooth bud.

5. Absence of space loss in extraction area.

6. Presence of the SM during study period.

7. Commitment of the patient to regular follow up visits. ${ }^{12,13}$

\section{Exclusion Criteria}

1. Presence of any systemic and mental disease.

2. Presence of mouth breathing habit.

3. Patients with high caries activity. ${ }^{12,13}$

Signed consent forms were collected from parents prior to the commencement of the study. Before cementing the appliances in place, we instructed proper dental hygiene to the patients and their parents and dental prophylaxis was performed. In the upper jaw palatal bar and in the lower jaw lingual - arch SMs were cemented. The bands of SMs were selected (3M Unitek, St. Paul, MN, USA), impressions were taken and SMs were fabricated in orthodontic laboratory. Fixed SMs were cemented by an orthodontist with a fluoride releasing glass ionomer cement (Rely X; 3M ESPE, St. Paul, MN, USA). Excess cement was removed using dental floss and scaler. For every selected child, the clinical examinations were done by a trained orthodontist for evaluation of DMFT, bleeding on probing, clinical attachment level, gingival index, and periodontal pocket depth at the baseline immediately before SM placement (T0), one month (T1), three months (T2) and six months (T3) after SM placement. All examinations were performed by the same orthodontist. A separate form was prepared for each session so the clinician was blind to the patients' previous data.

\section{DMFT}

DMFT was calculated for the banded teeth and for the entire jaw and recorded separately. DMFT is sum of the number of decayed, missing and filled teeth. ${ }^{14}$

\section{Bleeding on Probing (BOP)}

BOP was recorded using Muhlemann and Son index ${ }^{15}$ after gently moving the periodontal probe in $1 \mathrm{~mm}$ depth from distal interproximal of buccal sulcus to the mesial interproximal. BOP was recorded after 30 seconds. The procedure was repeated in palatal - lingual surface and BOP recorded that was defined as below -

- Score 0 gingiva of normal texture and colour, no bleeding;

- Score 1 - gingiva apparently normal, bleeding on probing;

- Score 2 - bleeding on probing, change in colour, no oedema;

- $\quad$ Score 3 - bleeding on probing, change in colour, slight oedema;

- Score 4 - either: (a) bleeding on probing, change in colour, obvious oedema; or (b) bleeding on probing, obvious oedema;

- Score 5 - bleeding on probing and spontaneous bleeding, change in colour, marked oedema. 


\section{Gingival Index (GI)}

It was used for the evaluation of inflammation severity in four areas of banded molars, gingiva including mesial and distal of buccal and lingual surfaces. GI was defined as below. ${ }^{16}$

- $\quad$ Score $0=$ Normal gingiva.

- Score 1 = Mild inflammation - slight change in colour, slight oedema. No bleeding on probing.

- Score 2 = Moderate inflammation - redness, oedema, glazing. Bleeding on probing.

- $\quad$ Score 3 = Severe inflammation - marked redness and oedema, ulceration. Tendency towards spontaneous bleeding.

\section{Periodontal Pocket Depth (PPD)}

Periodontal pocket depth was measured using a periodontal probe on buccal and lingual surface of first molar teeth in SM bearing jaw before band placement and in follow up visits and was recorded in millimetres.

\section{Clinical Attachment Level (CAL)}

It was measured as the distance between the cemento enamel junction and depth of probing using a periodontal probe and was reported in millimetres in buccal surface of first molars in SM bearing jaws. ${ }^{17}$

\section{Statistical Analysis}

The Kolmogorov - Smirnov test was applied to test for normal distribution. Comparisons of the parameters in the groups and times were evaluated according to repeated times variance analyses and two-way variance analyses DMFT and P $<0.05$ was set as level of significance.

\section{RESULTS}

A total of 20 patients who received fixed space maintainers, aged 8 - 11.7 years were included. DMFT values before SM placement and 1, 3 and 6 months after SM placement for the entire jaw and banded molars is shown in Table 1 separately. Before SM placement the Mean \pm SD of DMFT was $1.1 \pm 1$ and $0.2 \pm 0.6$ for the entire jaw except banded molars and for banded molars respectively.

Repeated measures ANOVA showed no significant differences between DMFT means of entire jaw and banded molars in time intervals (P-Value $=0.35$ and 0.163 respectively)

\begin{tabular}{|ccc|}
\hline $\begin{array}{c}\text { Evaluation } \\
\text { Time }\end{array}$ & $\begin{array}{c}\text { Entire Jaw Except Banded } \\
\text { Molars DMFT (Mean } \pm \text { SD) }\end{array}$ & $\begin{array}{c}\text { Banded Molars DMFT } \\
\text { ( Mean } \pm \text { SD) }\end{array}$ \\
$\mathrm{T}_{0}$ & $1.1 \pm 1$ & $0.2 \pm 0.6$ \\
$\mathrm{~T}_{1}$ & $1.1 \pm 1$ & $0.2 \pm 0.6$ \\
$\mathrm{~T}_{2}$ & $1.1 \pm 1$ & $0.2 \pm 0.6$ \\
$\mathrm{~T}_{3}$ & $1.1 \pm 1$ & $0.2 \pm 0.8$ \\
\hline \multicolumn{3}{|c|}{ Table 1. DMFT Values in Different Time Values } \\
\hline
\end{tabular}

Mean \pm SD of periodontal pocket depth (PPD) in buccal and lingual surface of banded molars were measured in millimetres $\left(\mathrm{T}_{0}=2 \pm 0.3, \mathrm{~T}_{1}=2.6 \pm 0.5, \mathrm{~T}_{2}=2.9 \pm 0.2\right.$ and $\mathrm{T}_{4}=3$ \pm 0 in buccal surface and $\mathrm{T}_{0}=1.8 \pm 0.6, \mathrm{~T}_{1}=2.6 \pm 0.5, \mathrm{~T}_{2}=2.9 \pm$
0.3 and $\mathrm{T}_{4}=3.4 \pm 0.5$ in lingual surface) and the data showed significant difference between time points, using repeated measures ANOVA (P-value < 0.001). To determine the difference between each interval, Post - Hoc LSD test was used and the results are shown in Table 2. Probing depth significantly increased in each interval, except for T2 and T3 interval in buccal surface.

\begin{tabular}{|ccccc|}
\hline Time Points & \multicolumn{2}{c|}{ Mean Difference(mm) } & \multicolumn{2}{c|}{ P - Value } \\
Buccal & Lingual & Buccal & Lingual \\
$\mathrm{T}_{0}$ and $\mathrm{T}_{1}$ & 0.6 & 0.8 & $<0.001^{*}$ & $<0.001^{*}$ \\
$\mathrm{~T}_{0}$ and $\mathrm{T}_{2}$ & 0.9 & 1 & $<0.001^{*}$ & $<0.001^{*}$ \\
$\mathrm{~T}_{0}$ and $\mathrm{T}_{3}$ & 1 & 1.5 & $<0.001^{*}$ & $<0.001^{*}$ \\
$\mathrm{~T}_{1}$ and $\mathrm{T}_{2}$ & 0.3 & 0.3 & $<0.001^{*}$ & $0.001^{*}$ \\
$\mathrm{~T}_{1}$ and $\mathrm{T}_{3}$ & 0.4 & 0.7 & $<0.001^{*}$ & $<0.001^{*}$ \\
$\mathrm{~T}_{2}$ and $\mathrm{T}_{3}$ & 0.05 & 0.5 & 0.160 & $<0.001^{*}$ \\
\hline \multicolumn{5}{c}{ Table 2. Mean Difference of PPD in Different Time Points, } \\
Results of LSD Test \\
*statistically significant changes \\
\hline \multicolumn{5}{c}{} \\
\hline
\end{tabular}

\begin{tabular}{|ccc|}
\hline Time Points & Mean Difference & P - Value \\
$\mathrm{T}_{0}$ and $\mathrm{T}_{1}$ & 0.6 & $<0.001^{*}$ \\
$\mathrm{~T}_{0}$ and $\mathrm{T}_{2}$ & 0.9 & $<0.001^{*}$ \\
$\mathrm{~T}_{0}$ and $\mathrm{T}_{3}$ & 1 & $<0.001^{*}$ \\
$\mathrm{~T}_{1}$ and $\mathrm{T}_{2}$ & 0.3 & $<0.001^{*}$ \\
$\mathrm{~T}_{1}$ and $\mathrm{T}_{3}$ & 0.4 & $<0.001^{*}$ \\
$\mathrm{~T}_{2}$ and $\mathrm{T}_{3}$ & 0.05 & $<0.001^{*}$ \\
\hline \multicolumn{2}{|c|}{ Table 3. Mean Difference of CAL in Different Time Points, } \\
Results of LSD Test \\
*statistically significant changes
\end{tabular}

\begin{tabular}{|ccccc|}
\hline Time Points & \multicolumn{2}{c|}{ Mean Difference } & \multicolumn{2}{c|}{ P - Value } \\
Buccal & Lingual & Buccal & Lingual \\
$\mathrm{T}_{0}$ and $\mathrm{T}_{1}$ & 0.6 & 0.7 & $<0.001^{*}$ & $<0.001^{*}$ \\
$\mathrm{~T}_{0}$ and $\mathrm{T}_{2}$ & 0.1 & 0.6 & $<0.001^{*}$ & $<0.001^{*}$ \\
$\mathrm{~T}_{0}$ and $\mathrm{T}_{3}$ & 0.5 & 0.9 & $<0.001^{*}$ & $<0.001^{*}$ \\
$\mathrm{~T}_{1}$ and $\mathrm{T}_{2}$ & 0.4 & -0.05 & $0.002^{*}$ & 0.568 \\
$\mathrm{~T}_{2}$ and $\mathrm{T}_{3}$ & -0.5 & 0.3 & $<0.001^{*}$ & $0.001^{*}$ \\
\hline Table 4. Mean Difference of BOP between Different Time Points, \\
Results of LSD Test \\
*statistically significant changes
\end{tabular}

\begin{tabular}{|ccccc|}
\hline \multirow{2}{*}{ Time Points } & \multicolumn{4}{c|}{ Number (Percentage) of the Teeth } \\
& $4(10 \%)$ & $26(65 \%)$ & $10(25 \%)$ & 0 \\
$\mathrm{~T}_{0}$ & $4(10 \%)$ & $24(60 \%)$ & $12(30 \%)$ & 0 \\
$\mathrm{~T}_{1}$ & $2(5 \%)$ & $20(50 \%)$ & $14(35 \%)$ & $2(5 \%)$ \\
$\mathrm{T}_{2}$ & $2(5 \%)$ & $10(25 \%)$ & $24(60 \%)$ & $4(10 \%)$ \\
$\mathrm{T}_{3}$ & \multicolumn{4}{|c|}{ Table 5. The Number (Percentages) of the Teeth Based on GI } \\
\hline
\end{tabular}

To compare clinical attachment levels (CAL) repeated measures ANOVA was used and there was significant difference between CAL between time points (P-value < 0.001). Table 3 shows differences between intervals using Post - Hoc LSD test. Bleeding on Probing in buccal and lingual surfaces of banded teeth, showed significant changes between time points using repeated measures ANOVA (P-value < 0.001). Post - hoc LSD test results to determine the differences between each interval is shown in Table 4 which indicates that there is significant increase in BOP before SM placement and each time point. Gingival Index increased significantly between intervals which was evaluated by friedman test. Table 5 (P value $<0.001$ ).

\section{DISCUSSION}

Fixed SMs are widely used in dental practice. But the longstanding presence of foreign bodies like fixed prosthesis and orthodontic appliances rise the suspicion of worsening gingival and dental parameters. ${ }^{5,18-20}$ The accumulation of 
dental biofilm around the bands and wires can cause detrimental effects in contagious tissues. ${ }^{21}$

In the present study twenty young children in mixed dentition period were selected. Each patient had two first molars that went under band for fixed space maintainer. The patients were examined for DMFT, bleeding on probing, clinical attachment level, gingival index, and periodontal pocket depth at the placement time of the SMs at one, three and six months later. In the present study we evaluated the effect of placement of fixed SMs for the first time. There was no significant increase in DMFT before and 6 months after SM placement. These results were consistent with the results of the Chan and Zhou's study which claimed that there was no significant difference in DMFT of patients with history of fixed orthodontic treatment and without it. ${ }^{22}$ Topaloglu et al. showed that Streptococcus Mutans increased significantly 6 months after the placement of fixed or removable orthodontic appliances, which can induce caries and increase DMFT,23 However one cannot expand the results of studies on full fixed orthodontic appliance over a simple band and loop appliances. The other reason for this controversy could be attributed to the difference in the mean age of the patients of these studies, because we know that oral microflora and caries susceptibility can change with aging. ${ }^{24}$ On the other hand keeping in mind that although the number of cariogenic biofilms as a caries risk factor increases during fixed orthodontic treatment, caries development is a long term procedure, so it's reasonable that interval of more than 6 months is mandatory to anticipate any change in DMFT.

Fixed orthodontic appliances are associated with increased accumulation of biofilm due to difficulty in oral hygiene maintenance.25 It has been indicated that periodontopathic and superinfecting bacteria increase during fixed orthodontic treatment, which could lead to inflammation of the periodontal tissues. ${ }^{26}$ Inflammation of gingival and periodontal tissues are indicated by Gingival index and Bleeding on probing index. ${ }^{27}$ In the present study, BOP and GI increased significantly after SM placement. Ristic et al. claimed that these periodontal index values increased during fixed orthodontic treatment which is consistent with results of the present study. 28

Periodontal pocket depth was significantly greater after SM placement which is in line with findings of Karkhanechi et al. ${ }^{29}$ Also Ahmed et al. showed that pocket depth increases in teeth with orthodontic bands which might be a result of inflammation induced by biofilm accumulation and overhangs of orthodontic bands, ${ }^{21}$ or more penetration depth of periodontal probe into weakened connective tissue. ${ }^{20}$ These factors also may cause loss of clinical attachment level. The question is that why periodontal breakdown occurs, but caries does not increase at the same time. It can be attributed to the nature of the soft tissues versus hard tissues of the teeth, and also the properties of different microorganisms that cause them.

In contrast with other periodontal indices reported here, clinical attachment loss can be assumed as a permanent effect of fixed $\mathrm{SMs}^{30}$ and is of great importance in the future health of the periodontium. The Gold Standard of periodontal health status measurement in patients with fixed orthodontics, is evaluation of the extent of clinical attachment loss. ${ }^{21}$ As we said previously the studies on full fixed appliances is not the same as on simple SMs so it was necessary to evaluate the detrimental effects on CAL in fixed SMs that we did it for the first time. 5,20 In the present study the amount of clinical attachment loss was significant after SM placement and this finding should warn the clinician to be more vigilant in case selection for SMs and oral hygiene supervision.

\section{CONCLUSIONS}

There was no significant difference in DMFT before and after SM placement. Bleeding on probing, Gingival index and periodontal pocket depth increased significantly after SM placement. Significant clinical attachment loss occurred after SM placement. The fixed space maintainers might have some detrimental effects on the periodontal structures of the banded teeth, so the clinicians should be more cautious of their potential harms to the teeth and periodontium for best management of these appliances and the patients must be under proper oral hygiene control and regular check-ups. The potential effect of these appliances on caries formation needs more studies.

\section{Recommendations}

We strongly recommend conducting a long-term study with matched cases to evaluate the effect of fixed SMs on DMFT of the patients.

Data sharing statement provided by the authors is available with the full text of this article at jemds.com.

Financial or other competing interests: None.

Disclosure forms provided by the authors are available with the full text of this article at jemds.com.

Authors' Contributions - Conception / Design of the study: AB, AP and MA. Data acquisition: ZA, VA, PN and MKM. Data analysis or interpretation: MKM. Writing the article: PN. Critical revision of the article: AB. Final approval of the article: AB, PN, ZA, VA, MKM, MA, AP. Overall responsibility: $\mathrm{AB}, \mathrm{PN}$.

\section{REFERENCES}

[1] Choonara SA. Orthodontic space maintenance--a review of current concepts and methods. SADJ 2005;60(3):1137.

[2] Watt E, Ahmad A, Adamji R, et al. Space maintainers in the primary and mixed dentition-a clinical guide. Br Dent J 2018;225(4):293-8.

[3] Law C. Management of premature primary tooth loss in the child patient. J Calif Dent Assoc 2013;41(8):612-8.

[4] Klukowska M, Bader A, Erbe C, et al. Plaque levels of patients with fixed orthodontic appliances measured by digital plaque image analysis. Am J Orthod Dentofacial Orthop 2011;139(5):e463-70.

[5] Richter AE, Arruda AO, Peters MC, et al. Incidence of caries lesions among patients treated with comprehensive orthodontics. Am J Orthod Dentofacial Orthop 2011;139(5):657-64.

[6] Cantekin K, Celikoglu M, Karadas M, et al. Effects of orthodontic treatment with fixed appliances on oral 
health status: a comprehensive study. J Dent Sci 2011;6(4):235-8.

[7] Baka ZM, Basciftci FA, Arslan U. Effects of 2 bracket and ligation types on plaque retention: a quantitative microbiologic analysis with real-time polymerase chain reaction. Am J Orthod Dentofacial Orthop 2013;144(2):260-7.

[8] Kim K, Heimisdottir K, Gebauer U, et al. Clinical and microbiological findings at sites treated with orthodontic fixed appliances in adolescents. Am J Orthod Dentofacial Orthop 2010;137(2):223-8.

[9] Batoni G, Pardini M, Giannotti A, et al. Effect of removable orthodontic appliances on oral colonisation by mutans streptococci in children. Eur J Oral Sci 2001;109(6):38892.

[10] Jordan C, LeBlanc DJ. Influences of orthodontic appliances on oral populations of mutans streptococci. Oral Microbiol Immunol 2002;17(2):65-71.

[11] Lau PYW, Wong RWK. Risks and complications in orthodontic treatment. Hong Kong Dent J 2006;3(1):15.

[12] Arikan V, Kizilci E, Ozalp N, et al. Effects of fixed and removable space maintainers on plaque accumulation, periodontal health, candidal and enterococcus faecalis carriage. Med Princ Pract 2015;24(4):311-7.

[13] Arikan F, Eronat N, Candan U, et al. Periodontal conditions associated with space maintainers following two different dental health education techniques. J Clin Pediatr Dent 2007;31(4):229-34.

[14] Cypriano S, De Sousa MDLR, Wada RS. Avaliação de índices CPOD simplificados em levantamentos epidemiológicos de cárie dentária. Rev Saude Publica 2005;39(2):285-92.

[15] Muhlemann HR, Son S. Gingival sulcus bleeding--a leading symptom in initial gingivitis. Helv Odontol Acta 1971;15(2):107-13.

[16] Löe H. The gingival index, the plaque index and the retention index systems. J Periodontol 1967;38(6):610-6.

[17] Rheu GB, Ji S, Ryu JJ, et al. Risk assessment for clinical attachment loss of periodontal tissue in Korean adults. J Adv Prosthodont 2011;3(1):25-32.

[18] De Backer H, Van Maele G, De Moor N, et al. A 20-year retrospective survival study of fixed partial dentures. Int J Prosthodont 2006;19(2):143-53.
[19] Knoernschild KL, Campbell SD. Periodontal tissue responses after insertion of artificial crowns and fixed partial dentures. J Prosthet Dent 2000;84(5):492-8.

[20] Davis SM, Plonka AB, Fulks BA, et al. Consequences of orthodontic treatment on periodontal health: clinical and microbial effects. Semin Orthod 2014;20(3):139-49.

[21] Ahmed I, Saif ul H, Nazir R. Periodontal status of first molars during orthodontic treatment. J Ayub Med Coll Abbottabad 2011;23(1):55-7.

[22] Chen W, Zhou Y. Caries outcomes after orthodontic treatment with fixed appliances: a longitudinal prospective study. Int J Clin Exp Med 2015;8(2):2815-22.

[23] Topaloglu-Ak A, Ertugrul F, Eden E, et al. Effect of orthodontic appliances on oral microbiota- 6 month follow-up. J Clin Pediatr Dent 2011;35(4):433-6.

[24] Sampaio-Maia B, Monteiro-Silva F. Acquisition and maturation of oral microbiome throughout childhood: an update. Dent Res J (Isfahan) 2014;11(3):291-301.

[25] Lombardo L, Ortan YÖ, Gorgun Ö, et al. Changes in the oral environment after placement of lingual and labial orthodontic appliances. Prog Orthod 2013;14(1):28.

[26] Naranjo AA, Triviño ML, Jaramillo A, et al. Changes in the subgingival microbiota and periodontal parameters before and 3 months after bracket placement. Am J Orthod Dentofacial Orthop 2006;130(3):275.e17-22.

[27] Lang NP, Joss A, Orsanic T, et al. Bleeding on probing. A predictor for the progression of periodontal disease? J Clin Periodontol 1986;13(6):590-6.

[28] Ristic M, Svabic MV, Sasic M, et al. Clinical and microbiological effects of fixed orthodontic appliances on periodontal tissues in adolescents. Orthod Craniofac Res 2007;10(4):187-95.

[29] Karkhanechi M, Chow D, Sipkin J, et al. Periodontal status of adult patients treated with fixed buccal appliances and removable aligners over one year of active orthodontic therapy. Angle Orthod 2013;83(1):146-51.

[30] Brennan RM, Genco RJ, Hovey KM, et al. Clinical attachment loss, systemic bone density and subgingival calculus in postmenopausal women. J Periodontol 2007;78(11):2104-11. 